\title{
Diverse expression patterns and tumorigenic role of neurotensin signaling components in colorectal cancer cells
}

\author{
JI TAE KIM ${ }^{1}$, HEIDI L. WEISS ${ }^{1}$ and B. MARK EVERS ${ }^{1,2}$ \\ ${ }^{1}$ Markey Cancer Center, University of Kentucky; ${ }^{2}$ Department of Surgery, University of Kentucky, \\ Lexington, KY 40536, USA
}

Received January 27, 2017; Accepted May 2, 2017

DOI: 10.3892/ijo.2017.3990

\begin{abstract}
Colorectal cancer (CRC), which is one of the most common malignancies worldwide, results from an accumulation of genetic and epigenetic modifications including DNA methylation. Neurotensin (NTS), a hormone localized to the gut and central nervous system, mediates its physiological and pathological effects, including growth stimulation for a variety of cancers, through three distinct NTS receptors (NTSRs). Most NTS functions are mediated through the high-affinity receptor NTSR1, and expression of NTSR1 is increased in many cancers including CRC. In this study, we investigated the expression profiles and cellular functions of the NTSRs, especially NTSR 1 , in CRC cells. We showed that expression levels for NTS and NTSR 1 varied, that NTSR 2 expression was not detectable and that NTSR 3 was consistently expressed in all CRC cell lines examined. Treatment with the demethylating agent, 5-aza-2'-deoxycytidine, augmented levels of NTSR1/2 in Caco2 and DLD1 cells, which have little or no transcripts for NTSR $1 / 2$ suggesting that DNA methylation suppresses NTSR1/2 expression. In addition, we demonstrated that knockdown of NTSR1 decreased cell growth and migration in HCT116 and HT29 cells. Finally, we showed that treatment with SR48692, an antagonist of NTSR1, also inhibited cell proliferation and migration in the CRC cells. Our findings identify promoter methylation as an important process regulating the differential expression or silencing of NTSR1/2 in CRC cells. Moreover, inhibition of NTSR1
\end{abstract}

Correspondence to: Dr B. Mark Evers, Markey Cancer Center, University of Kentucky, 800 Rose Street, CC140 Ben F. Roach Bldg., Lexington, KY 40536-0093, USA

E-mail: mark.evers@uky.edu

Abbreviations: CRC, colorectal cancer; NTS, neurotensin; NTSR, neurotensin receptor; GI, gastrointestinal; NET, neuroendocrine tumor; FBS, fetal bovine serum; 5-aza-CdR, 5-aza-2'-deoxycytidine; RT-(q)PCR, (quantitative) reverse transcription-polymerase chain reaction; MSP, methylation-specific PCR; BS, bisulfite sequencing; IL-8, interleukin-8

Key words: CRC cells, neurotensin receptor, promoter methylation, cell growth, migration repressed tumorigenic effects in CRC cells, suggesting that NTSR1 may be used as a therapeutic target for CRC.

\section{Introduction}

Colorectal cancer (CRC) is one of the most common malignancies in the United States and worldwide (1). Genetic alterations, specifically gene mutations implicated in CRC tumorigenesis, lead to a gain of oncogene function and loss of tumor suppressor gene function (2). Epigenetic modifications, particularly DNA methylation in selected gene promoters, are also recognized common molecular alterations in human tumors $(2,3)$.

Neurotensin (NTS), a tridecapeptide mainly distributed along the gastrointestinal (GI) tract, functions to decrease gastric motility, to increase pancreaticobiliary secretion, to facilitate fatty acid absorption, and to increase proliferation of normal intestinal mucosa (4-6). In addition to these physiologic effects, NTS influences intestinal inflammation and promotes the growth of cancers, including breast, prostate, pancreas, lung and cancers of the GI tract $(4,7,8)$. The effects of NTS are exerted primarily by its G protein-coupled receptors: the high-affinity NTS receptor 1 (NTSR1), and the lowaffinity NTSR $2(7,8)$. NTSR3/sortilin, a single transmembrane receptor, also binds NTS and contributes to the diversity of effects on multiple tissues $(7,8)$.

The properties of NTS are predominantly mediated through NTSR1, and this neuropeptide-receptor complex is deregulated during cancer progression $(8,9)$. Although increased expression of NTSR1 has been identified in certain cancer types, such as colon and neuroendocrine tumors (NETs) (10-16), the molecular mechanisms regulating NTSRs expression have not been clarified. Recently, we reported that promoter methylation is an important molecular process that regulates the differential expression of NTSR 1 and silences NTSR 2 in NET cells, and that silencing of NTSR1 suppressed the oncogenic effects of NTS (16). In the current study, we analyzed the endogenous expression of NTS signaling components, the transcriptional change of NTSR genes mediated by a demethylating agent, and the methylation status of their promoters in CRC cell lines. Importantly, we show that inhibition of NTSR1 by either gene knockdown or treatment with an NTSR1 inhibitor decreases the growth and migration of CRC cells. 


\section{Materials and methods}

Cell lines, reagents and siRNA transfection. The human CRC cell lines KM12c, Caco2, DLD1, HT29, HCT116, and SW480 were used in these studies. Cell lines were authenticated in February and May 2016 at Genetica DNA Laboratories (Cincinnati, OH). KM12c cells were kindly provided by Dr Isaiah J. Fidler (M.D. Anderson Cancer Center, Houston, TX, USA); other cell lines were obtained from American Type Culture Collection (Manassas, VA, USA). KM12c cells were cultured in MEM supplemented with $10 \%$ fetal bovine serum (FBS), 1\% sodium pyruvate, $1 \%$ non-essential amino acids and 2\% MEM essential vitamins. Caco 2 cells were incubated in MEM supplemented with $15 \%$ FBS, $1 \%$ sodium pyruvate and $1 \%$ non-essential amino acids. DLD1 cells were grown in RPMI-1640 with $10 \%$ FBS. HT29 and HCT116 cells were maintained in McCoy's 5A medium supplemented with 10\% FBS. SW480 cells were cultured in DMEM with $10 \%$ FBS. Cells were maintained at $37^{\circ} \mathrm{C}$ in a humidified $5 \% \mathrm{CO}_{2}$ incubator. The DNA methyltransferase inhibitor, 5-aza-2'-deoxycytidine (5-aza-CdR), and a selective inhibitor for NTSR1, SR48692, were purchased from Sigma-Aldrich (St. Louis, MO, USA) and dissolved in dimethyl sulfoxide (DMSO). Transfections with non-targeting control and SMARTPool NTSR1 siRNA (Dharmacon, Lafayette, CO, USA) were performed using Lipofectamine RNAiMAX (Invitrogen, Carlsbad, CA, USA) as previously described (16).

RNA isolation, reverse transcription-PCR (RT-PCR) and quantitative reverse transcription- $P C R(R T-q P C R)$ analysis. Total RNA isolation, cDNA synthesis and RT-PCR analysis were carried out as previously described $(16,17)$. Briefly, RT-PCR reactions for NTS signaling elements were performed using cDNA synthesized from $1 \mu \mathrm{g}$ of total RNA from CRC cells, HotStarTaq DNA Polymerase (Qiagen, Valencia, CA, USA) and primers as follows: NTSR 1 F, 5'-TCATCGC CTTTGTGGTCTGCT-3', and NTSR1 R, 5'-TGGTTGCT GGACACGCTGTCG-3', 33 cycles; NTSR2 F, 5'-GTCTCC TCAGCTTCATCGTAT-3', and NTSR2 R, 5'-TCCCCAA AGCCTGAAGCTGTA-3', 40 cycles; NTSR3 F, 5'-AGAA TGGTCGAGACTATGTTG-3', and NTSR3 R, 5'-AAG AGCTATTCCAAGAGGTCC-5', 33 cycles; NTS F, 5'-GAT GATGGCAGGAATGAAAATCCAG-3', and NTS R, 5'-GTT GAAAAGCCCTGCTGTGACAGA-3', 40 cycles; $\beta$-actin F, 5'-TCACCAACTGGGACGACATG-3', and $\beta$-actin R, 5'-ACCGGAGTCCATCACGATG-3', 28 cycles; IL-8 F, 5'-CATGACTTCCAAGCTGGCCG-3', and $I L-8$ R, 5'-AAT TTTTTTATGAATTCTCAGCCCTC-3', 33 cycles; cyclin D1 F, 5'-ATGTGTGCAGAAGGAGGTCC-3' and cyclin DI R, 5'-CTTAGAGGCCACGAACATGC-3', 38 cycles. Cycling conditions for the reactions were: initial melting at $95^{\circ} \mathrm{C}$ for $15 \mathrm{~min}$, followed by the above described numbers of cycles at $94^{\circ} \mathrm{C}$ for $30 \mathrm{sec}, 55^{\circ} \mathrm{C}$ for $30 \mathrm{sec}$ and $72^{\circ} \mathrm{C}$ for $45 \mathrm{sec}$ and a final extension of $10 \mathrm{~min}$ at $72^{\circ} \mathrm{C}$ (MJ Mini Thermal Cycler, Bio-Rad, Irvine, CA, USA). The PCR products were analyzed on a $2 \%$ agarose gel and visualized with the Alpha Innotech Imaging system (Alpha Innotech Corp., San Leandro, CA, USA). RT-qPCR was carried out using TaqMan kits (Applied Biosystems, Foster City, CA, USA) under StepOnePlus
Real-Time PCR System (Applied Biosystems) as previously described (16), according to the manufacturer's protocol.

Methylation analysis. The methylation status of the NTSRI and NTSR 2 promoters was determined by methylation-specific PCR (MSP) and bisulfite sequencing (BS) analyses as previously described $(16,17)$. In brief, PCR with 35 cycling reactions was performed using bisulfite-modified genomic DNA, HotStarTaq DNA Polymerase (Qiagen) and primers as follows: NTSR1 MSP methyl (M) F, 5'-TTGGAATTCGTGGTA AGC-3', and NTSR1 MSP M R, 5'-GTCTCAAACGAAA ACCGATA-3'; NTSR1 MSP unmethyl (U) F, 5'-TATTTG GAATTTGTGGTAAGT-3', and NTSR1 MSP U R, 5'-ATCT CAAACAAAAACCAATAAAC-3'; NTSR2 MSP M F, 5'-GTGGAGTTCGGTTTAATTC-3', and NTSR2 MSP M R 5'-ACTACCCGAAATCTAAACG-3'; NTSR2 MSP U F, 5'-GGTGGAGTTTGGTTTAATTT-3', and NTSR2 MSP U R, 5'-CACTACCCAAAATCTAAACA-5'; NTSR1 BS F, 5'-TTGTGGATATTTAGGAGTGGG-3' and NTSR1 BS R, 5'-CTCCAAAAAACCAAAATTCC-3'; NTSR2 BS F, 5'-TGTTGGGAAAGTTTTTTTTAAG-3' and NTSR2 BS R, 5'-AAACACCTCCTCTTCTCTAAAAA-3'. The PCR products for MSP were visualized as indicated above. For BS, PCR products were cloned into the TOPO TA cloning vector (Invitrogen) and the plasmids from individual bacterial colonies were sequenced.

Cell proliferation. Equal numbers of HCT116 and HT29 cells were seeded in 24-well plates. Proliferation of cells transfected with siRNA or treated with SR48692 was assessed at 48 and $96 \mathrm{~h}$ after seeding by direct cell counting using a Beckman Coulter Cell Viability Analyzer (Beckman-Coulter, Fullerton, CA, USA).

Western blot analysis. Western blot analysis was done as previously described (16). The antibodies for rabbit polyclonal NTSR1 (PA3-214, 1:2500 dilution) and rabbit monoclonal cyclin D1 (2261-1, 1:5000 dilution) were purchased from Thermo Fisher Scientific (Rockford, IL, USA) and Epitomics (Burlingame, CA), respectively. The rabbit polyclonal IL-8 (ab106350, 1:500 dilution) and mouse monoclonal anti- $\beta$-actin (A5316, 1:5000 dilution) antibodies were obtained from Abcam (Cambridge, MA, USA) and Sigma-Aldrich, respectively.

Wound-healing migration assay. A wound-healing migration assay was performed, using the Ibidi Culture Insert (Ibidi, Munich, Germany), with control and NTSR1 knockdown HCT116 or HT29 cells. The wounded monolayers, generated by removal of the insert, which provides a cell-free gap, were maintained for the indicated time periods. Phase-contrast microscopic images were acquired using a Nikon Eclipse Ti microscope and NIS Elements software (Nikon, Melville, NY, USA). The data are the quantified gap distance.

Luciferase reporter assays. HCT116 and HT29 cells, seeded in 24-well plates, were transiently transfected with the IL-8 reporter $(0.4 \mu \mathrm{g})$ and the Renilla luciferase reporter $(0.05 \mu \mathrm{g})$ using Lipofectamine 2000 (Invitrogen) according to the manufacturers' instructions. For the NTSR1 inhibitor treatments, CRC cells were treated with SR48692 $(0$, or $10 \mu \mathrm{M})$ 


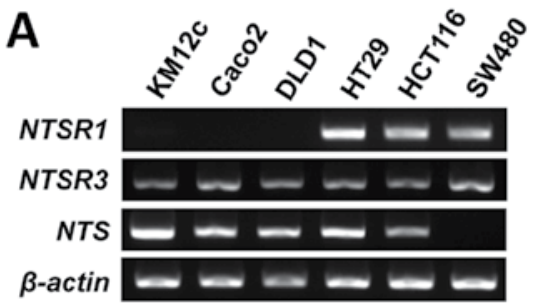

B

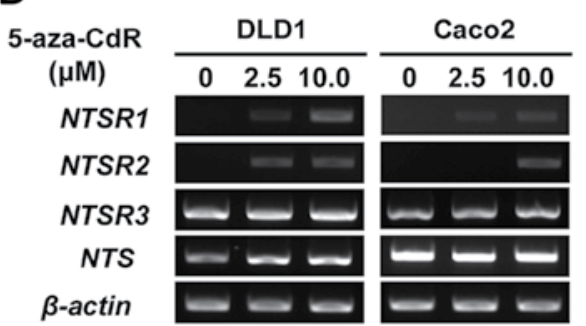

C

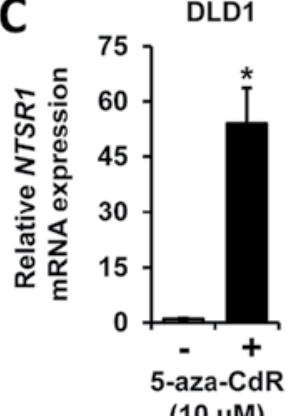

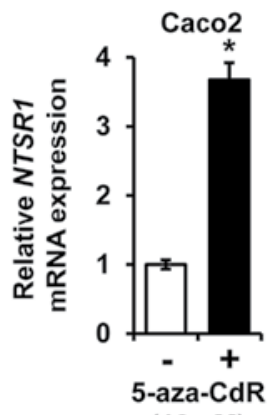

$(10 \mu \mathrm{M})$

Figure 1. Analysis of NTS signaling components in endogenous or 5-aza-CdR treated CRC cell lines. (A) Expression analysis of mRNA levels for NTS, NTSRs and $\beta$-actin in six CRC cells by RT-PCR. (B) Expression analysis of mRNA levels for NTS components and $\beta$-actin in DLD1 and Caco 2 cells treated with 0 , 2.5 or $10 \mu \mathrm{M} 5$-aza-CdR by RT-PCR. Media were replaced every $24 \mathrm{~h}$ for 4 days. (C) Quantitative RT-PCR analysis (RT-qPCR) for DLD1 and Caco2 cells treated with 5-aza-CdR. Presented data are the relative amounts of mRNA calculated by the $\Delta \Delta C q$ method performed in quadruplicate, with standard deviation (SD) shown. ${ }^{*} \mathrm{P}<0.05$ indicates a significant difference in mRNA levels between the inhibitor and vehicle $(0 \mu \mathrm{M})$ treatments; GAPDH was the internal control.

one day after transfection. Luciferase activity was measured from cell lysates using a Dual-Luciferase Reporter Assay System (Promega, Madison, WI) through Sirius Luminometer (Berthold Detection Systems, Pforzheim, Germany) according to the manufacturers' protocols.

Transwell migration assay. Cell migration assessments were performed using $8.0 \mu \mathrm{m}$ pore size Transwell filter inserts (Corning Inc., Corning, NY, USA) coated with $15 \mu \mathrm{g} / \mathrm{ml}$ type I collagen in 24-well plates. Briefly, HCT116 cells, serum-starved for $24 \mathrm{~h}$, were trypsinized, washed twice and resuspended in serum-free media supplemented with $0.1 \%$ BSA. For replicates, equivalent cell numbers were placed in the upper chamber, while the lower chamber was filled with the same serum-free media containing SR48692 (either 0 or $10 \mu \mathrm{M})$. The cells were incubated in the Transwell chambers for $20 \mathrm{~h}$ at $37^{\circ} \mathrm{C}$. After removing the non-migrated cells from the upper surface of the membrane, cells on the lower surface were fixed with methanol and stained with $0.5 \%$ crystal violet. Migration was determined by counting cell numbers in four random fields per membrane under a Nikon Eclipse 80i microscope and NIS Elements software (Nikon) at x10.

Statistical analysis. Means and standard deviations for triplicate or quadruple samples were calculated, and graphic representations summarize mRNA levels of NTSR 1 and NTSR2, number of counted cells, migration distance, IL-8 luciferase activity and migrated cell number. Comparisons between groups were performed using two-sample t-test or analysis of variance, as indicated, with tests for pairwise comparison or linear trend over dose levels.

\section{Results}

Expression analysis of NTS signaling components in endogenous or 5-aza-CdR treated CRC cell lines. To assess NTS and NTSR expression in CRC cells, we performed RT-PCR analysis of NTS constituents in six human CRC cell lines (KM12c, Caco2, DLD1, HT29, HCT116 and SW480). Whereas NTSR3 mRNA was consistently expressed in all six cell lines (Fig. 1A), NTSR2 expression was not observed (data not shown). Selective expression of NTSR 1 and NTS was noted; little to no expression of NTSR1 was noted in KM12c, Caco2 and DLD1 cells, and an absence of NTS expression was detected in SW480 cells (Fig. 1A). Recently, we showed that promoter methylation is a key mechanism regulating the differential expression of NTSR 1 and to silence NTSR2 in NET cells (16). To confirm whether the altered expression of NTSR1 and NTSR 2 in CRC cells was a result of DNA methylation, we treated DLD1 and Caco2 cells with the DNA methyltransferase inhibitor, 5-aza$\mathrm{CdR}$, and evaluated the expression of the NTSRs by RT-PCR (Fig. 1B). Treatment with 5-aza-CdR augmented the expression of NTSR1 and NTSR2 in DLD1 and Caco2 cells, respectively. To verify these results, the level of mRNA expression was also examined by RT-qPCR (Fig. 1C). Treatment with 5-aza-CdR resulted in an approximate 54-fold induction of NTSR1 expression in DLD1 and an approximate 4-fold induction in Caco2 cells. Together, these results suggest that NTSR1 and NTSR2 are targets of methylation in CRC cells.

Correlation between gene silencing and promoter methylation of NTSR1 and NTSR 2 in CRC cells. To determine whether induction of NTSR 1 and NTSR 2 expression by 5 -aza-CdR was due to promoter methylation, we investigated the methylation status of $\mathrm{CpG}$ islands for these genes using methylationspecific PCR (MSP) and bisulfite sequencing. While the NTSR $1 / 2$ promoters were found to be almost completely methylated in KM12c, Caco2 and DLD1 cells, the NTSR1 promoter in HT29, HCT116 and SW480 cells was shown to be unmethylated (Fig. 2A). The methylation profile of the $\mathrm{CpG}$ sites of NTSRl was further analyzed by bisulfite sequencing (Fig. 2B). Consistent with the MSP data, the CpG islands of NTSR1 were not methylated in HT29, HCT116 and SW480 cells. Moreover, promoter methylation of NTSR2 was noted in all six CRC cell lines by MSP analysis (Fig. 2A) and bisulfite sequencing analyses (Fig. 2C). These data demonstrate that promoter methylation silences the NTSR $1 / 2$ genes.

Knockdown of NTSR1 represses cell growth and migration in CRC cells. We previously reported that inhibition of NTS signaling components such as NTS and NTSR1 suppressed tumorigenic functions in NET cells $(16,18)$. To elucidate the potential oncogenic functions of NTS components in CRC cells, small interfering RNA (siRNA) directed against NTSRl, 
A

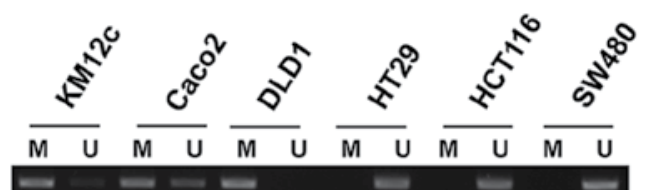

NTSR1

NTRS2

B

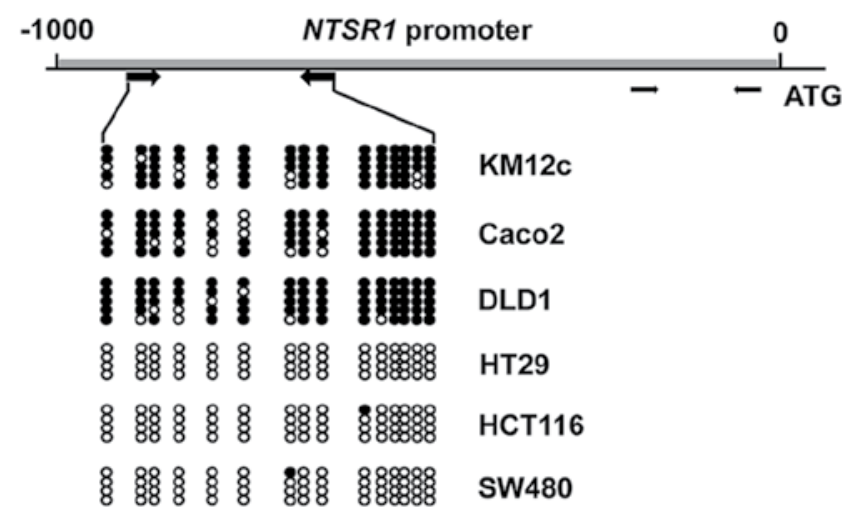

C

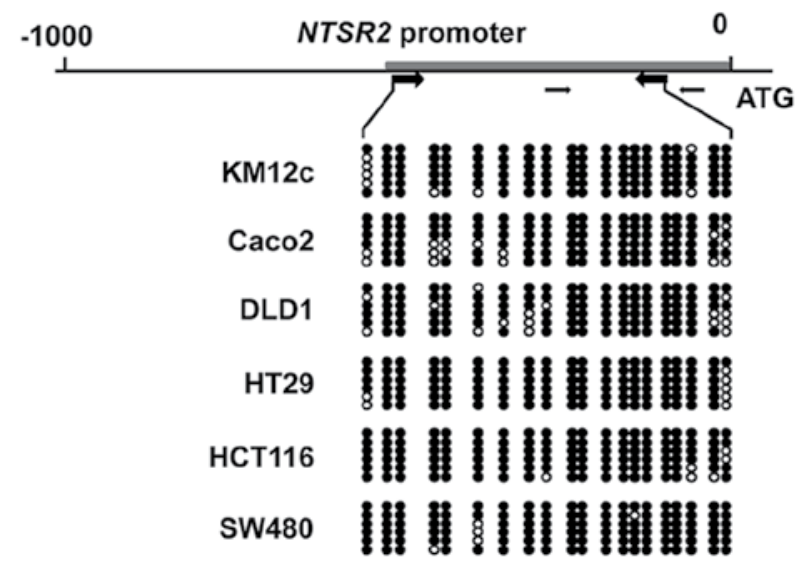

Figure 2. Methylation analysis of NTSR1 and NTSR2 promoter regions in CRC cells. (A) MSP analysis of the NTSR1 and NTSR2 promoters in six CRC cell lines using primers specific for methylated (M) and unmethylated (U) DNA, respectively. (B) Bisulfite genomic sequencing of NTSR1 CpG islands in CRC cell lines. Each row of circles indicates the DNA sequence of an individual clone; closed and open circles represent methylated and unmethylated CpG sites, respectively. Bold grey lines are candidate $\mathrm{CpG}$ islands detected by Methyl Primer Express Software v1.0 (Applied Biosystems). The arrows below the CpG islands represent sites of the primers for bisulfite sequencing (thicker) and MSP (thinner), respectively. (C) Bisulfite sequencing analysis of the 5' region of NTSR 2 in the CRC cells; protocol and data presentation are as outlined above.
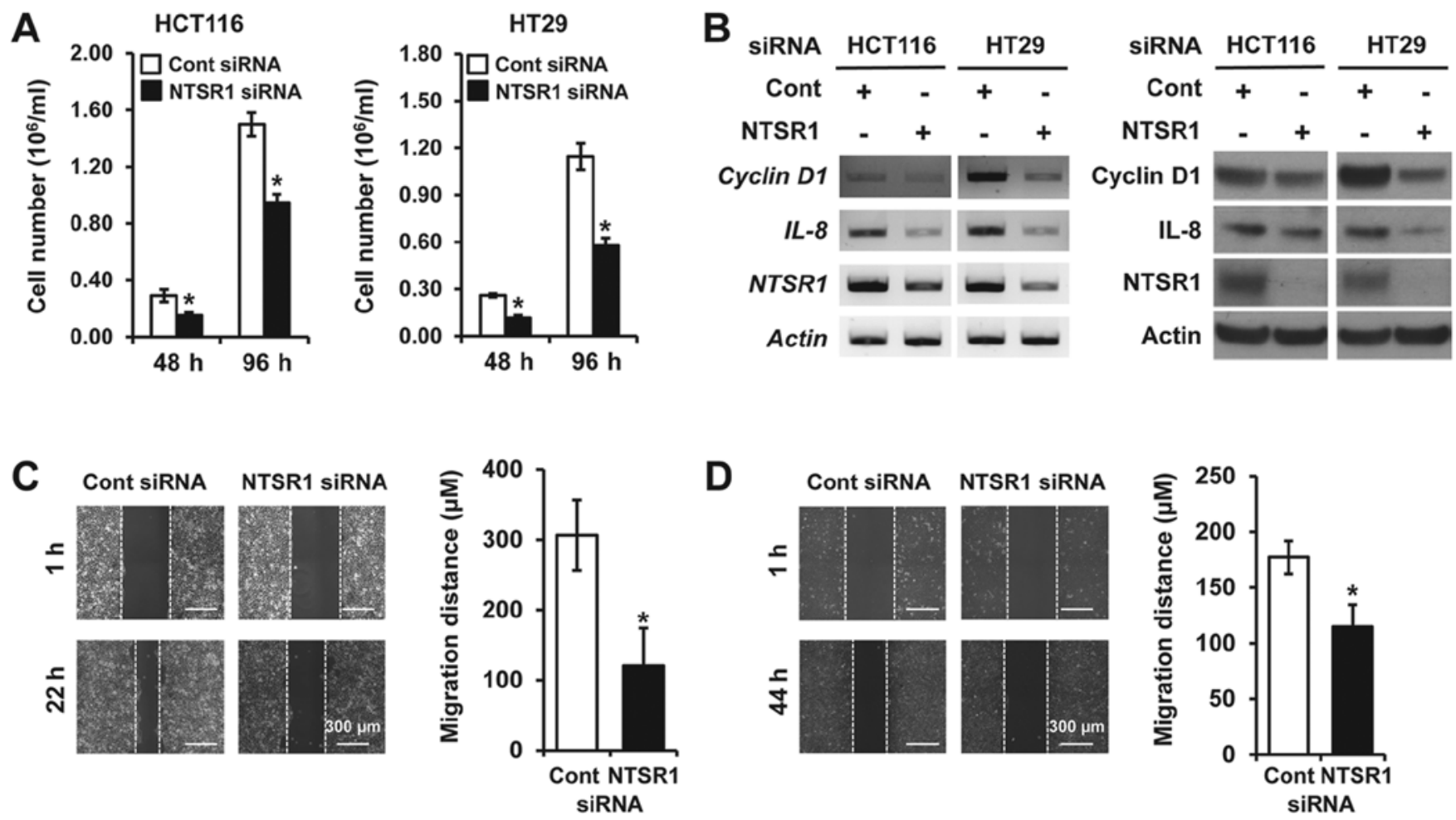

Figure 3. Silencing of NTSR1 influences cell growth and migration in CRC cells. (A) Equal numbers of HCT116 (left) and HT29 (right) cells transfected with siRNA against non-targeting (Cont) or NTSR 1 were seeded in 24 -well plates. Cells were counted after 48 and $96 \mathrm{~h}$ incubation using a cell counter. ${ }^{*} \mathrm{P}<0.05$ indicates a significant difference in cell number versus Cont. (B) Analyses of mRNA by RT-PCR (left) and protein by western blot (right) for expression of IL-8, cyclin D1 and NTSR1 in HCT116 and HT29 cells transfected with Cont or NTSR1 siRNA. $\beta$-actin was the loading control. (C) Migration analysis by wound-healing assay was performed with Cont and NTSR1 knockdown HCT116 cells. Phase-contrast microscopic images (left) show Cont or NTSR1-silenced cells at $1 \mathrm{~h}$ (upper) or $22 \mathrm{~h}$ (bottom) after removal of the insert (dark center/cell-free zone). White lines show the cell boundary and the graph (right) provides quantification of the migrated distance. ${ }^{*} \mathrm{P}<0.05$ indicates a significant difference in migration versus Cont. (D) Migration analysis with Cont and NTSR1 knockdown HT29 cells; protocol and data presentation are as outlined in (C). 
A

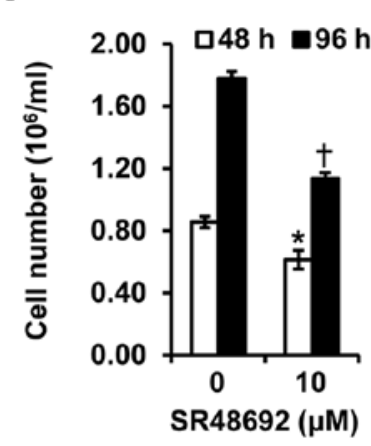

C

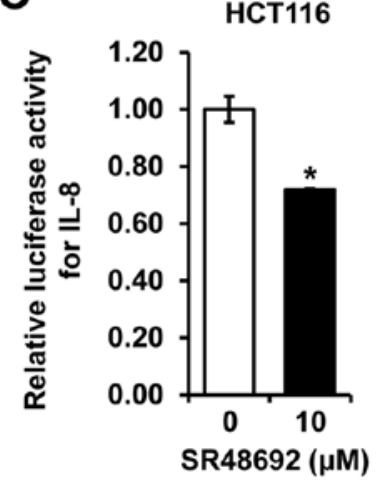

HT29

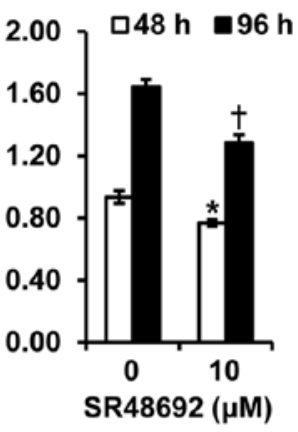

B

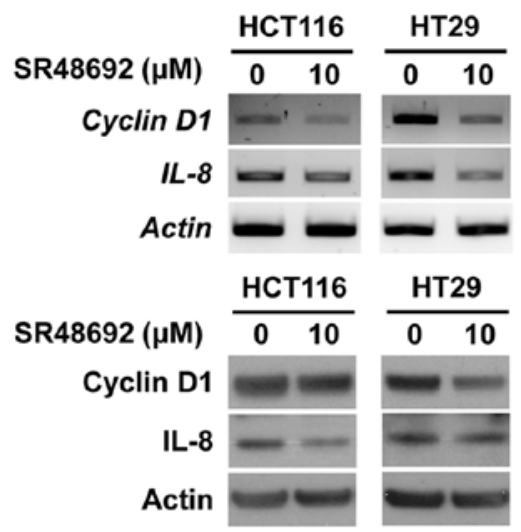

D

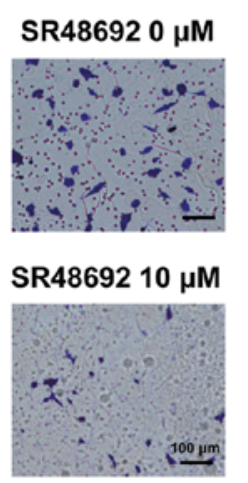

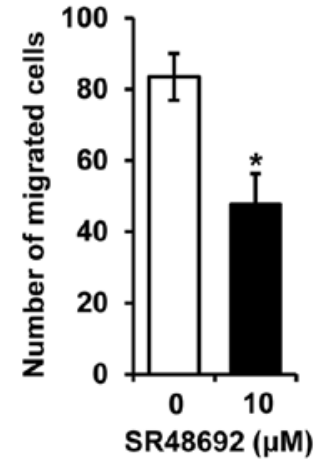

Figure 4. SR48692, an NTSR1 antagonist, decreases CRC cell growth and migration. (A) Equal numbers of HCT116 (left) and HT29 (right) cells were plated in 24-well plates. One day after seeding, cells were treated with 0 or $10 \mu \mathrm{M}$ SR48692 for 48 and 96 h; cell numbers were determined using a cell counter. Significant difference versus vehicle $(0 \mu \mathrm{M})$ treatments at $48 \mathrm{~h}(*)$ and $96 \mathrm{~h}(\dagger)$ are indicated, with P<0.05. (B) RT-PCR (upper) and western blot (bottom) analyses show regulated expressions of IL-8 and cyclin D1 in HCT116 and HT29 cells after SR48692 treatment for 2 days. (C) Graphic representation of the relative luciferase activity at the $I L-8$ promoter in HCT116 (left) and HT29 (right) cells treated with SR48692. * P $<0.05$ indicates a significant difference in IL-8 promoter activity versus vehicle treatment. (D) Migration analysis using the Boyden chamber with HCT116 cells treated with $10 \mu \mathrm{M}$ SR48692 or vehicle (control) for $20 \mathrm{~h}$. Phase-contrast microscopic images (left) and quantification of migrated cells observed in four different fields using an inverted microscope (right) are shown. ${ }^{*} \mathrm{P}<0.05$ indicates a significant difference in migrated cell number versus control.

which is inconsistently expressed among the tested cell lines (Fig. 1A), was used in HCT116 and HT29 cells since these cell lines do express the NTS transcript (Fig. 1A), and release NTS peptide (19). Knockdown of NTSR1 inhibited cell growth for 48 and $96 \mathrm{~h}$ in both HCT116 and HT29 cells compared with cells transfected with non-targeting control siRNA (Fig. 3A). Furthermore, silencing NTSR 1 significantly suppressed mRNA expression of $I L-8$, which can be induced by NTS signaling (20) and is associated with tumorigenic functions in CRC (21) and cyclin Dl, which is important for cell growth $(18,22)$ (Fig. 3B, left). Protein expressions of IL-8 and cyclin D1 were also decreased in cell lines with NTSR1 knockdown (Fig. 3B, right). In addition to cell proliferation, NTSR1 activation influences cell migration and invasion in some cancers including breast and NET $(13,16,23)$. On the basis of these studies, we next assessed the migratory activity of CRC cells transfected with NTSR1 siRNA using a wound-healing migration assay. Silencing NTSR1 suppressed cell migration of HCT116 cells (Fig. 3C) and HT29 cells (Fig. 3D), respectively. Collectively, these findings suggest that knockdown of NTSRl significantly inhibits the growth and migration of CRC cells.

SR48692, an NTSR1 antagonist, suppresses cell proliferation and migration of CRC cells. To further delineate the role of
NTSR1, we examined the effect of a pharmacologic blockade of NTSR1 using SR48692 in HCT116 and HT29 cells. Treatment with SR48692 repressed proliferation in HCT116 and HT29 cells for 48 and $96 \mathrm{~h}$ (Fig. 4A) and reduced both mRNA (upper panels) and protein expressions (lower panels) of IL-8 and cyclin D1 in the cell lines (Fig. 4B). Additionally, SR48692 inhibited the promoter activity of $I L-8$, which promotes tumor growth, metastasis and angiogenesis in CRC cell lines (21) (Fig. 4C). Moreover, HCT116 cells treated with SR48692 showed significantly decreased cell migration compared to vehicle-treated control cells (Fig. 4D). These results confirm that treatment with a selective NTSR1 antagonist inhibits cell growth and migration of CRC cells, and is consistent with results obtained with the siRNA experiments outlined above. These data indicate that inhibition of NTSR1, either through gene knockdown or receptor blockade, suppresses the growth and migration of CRC cells, and demonstrates a role for NTSR1 in CRC tumorigenesis.

\section{Discussion}

It has been reported that NTS and/or NTSR1 are overexpressed in some types of cancers and many cancer cell lines, and that inhibition of NTS signaling can suppress the oncogenic activities in several cancer cell lines $(10-14,16,23,24)$. Moreover, 
there is accumulating evidence that NTSR 1 activation is linked with poor prognosis, cancer progression and a higher incidence of metastases in lung and breast cancers $(13,14)$. However, the regulatory mechanism directing the expression of NTS pathway components in CRC cells is not well-delineated. One regulatory process has been proposed; activation of $\mathrm{Wnt} / \beta$-catenin signaling plays an essential role in the upregulation of NTSR1 in CRC (25) and NTS is a direct target of the Wnt/ $\beta$-catenin pathway in NET cells (18). In addition, Dong et al in our laboratory showed that promoter methylation contributes to the regulation of NTS expression in human cancer cells including colon cancer $(26,27)$ and we have also recently demonstrated that promoter methylation is an important molecular process regulating the expression of NTSR 1 and NTSR 2 in NET cells (16). Herein, we observed diverse expression of NTSRl and NTS, no expression of NTSR2, and consistent expression of NTSR 3 in the CRC cells examined in this study. In particular, we speculate that the varied level of NTS expression (KM12c, Caco2, DLD1, HT29 and HCT116 versus SW480 cells, Fig. 1A) is attributed to promoter methylation on the basis of our current result and previous study that DNA methylation is associated with NTS expression in CRC cells (27). We also found that the gene expression patterns for NTSR1 and NTSR 2 in CRC cells are similar to those in NET (16) suggesting that promoter methylation of NTS signaling constituents is closely associated with silencing of the genes in human cancer cells.

In NTS pathway genes (especially NTSR 1 and NTSR2) whose expression was demonstrated to be modulated by promoter methylation in our study, it has been reported that activation of NTSR1 induces cell proliferation, migration and invasion in a variety of cancers $(8,11,24)$. Therefore, we evaluated the role of NTSR1 on cell growth and migration in CRC cell lines. In previous studies, the effects of NTS-mediated NTSR1 activation were usually assessed through direct treatment of cancer cells with NTS or an NTS agonist $(11,15,23,24)$. Herein, we determined the effect of NTSR1 inhibition without NTS treatment on cell growth and migration in CRC cells Previously, NTS expression was reported in some cancer cells including CRC (11-14); these findings were confirmed in our present study by RT-PCR analysis (Fig. 1A). In addition, our group identified NTS peptide secretion in CRC cell lines including HCT116 and HT29 (19). Analogous to our previous findings and current study, we find that endogenous NTS can exert stimulatory properties of cell growth and migration through NTSR1 in CRC cells through both autocrine and paracrine pathways.

As aforementioned, it has been reported that a higher level of NTSR1 expression is observed in several human cancer tissues such as pancreas, lung, breast, prostate, colon and NET and that the NTSR1 correlates with tumor progression and aggressiveness (10-16). These findings suggest that NTSR1 may be a potential diagnostic biomarker and treatment target for these cancers. However, as noted above, NTSR1 promoter hypermethylation, which can lead to the gene silencing, was also found in lung and pancreatic cancers through genome scanning or global DNA methylation profiling, and was identified as a predictive marker for the cancers (28-30). These intricacies imply that additional unrevealed adjusters or mechanisms may affect the level of NTSR1 expression and that further work is needed to clarify the detailed machinery between expression and silencing of NTSR1 in various types of cancer.

In conclusion, our study shows that promoter methylation is a key regulatory event for NTSR 1 and NTSR 2 expression in CRC cells. In addition, we confirmed that inhibition of NTSR1 suppresses cell proliferation and migration in these cells. Our findings provide a rationale and incentive to explore NTSR 1 as a therapeutic target of $\mathrm{CRC}$ growth and pathogenesis.

\section{Acknowledgements}

We thank Catherine E. Anthony (Markey Cancer Center's Research Communications Office, University of Kentucky) for assistance with manuscript preparation. This study was supported by National Institutes of Health (NIH) grant R01 DK112034 and by the Biostatistical and Bioinformatics shared resource facility of the University of Kentucky Markey Cancer Center (supported by National Cancer Institute grant no. P30CA177558).

\section{References}

1. Siegel RL, Miller KD and Jemal A: Cancer statistics, 2015. CA Cancer J Clin 65: 5-29, 2015.

2. Fearon ER: Molecular genetics of colorectal cancer. Annu Rev Pathol 6: 479-507, 2011.

3. Ashktorab $\mathrm{H}$ and Brim $\mathrm{H}$ : DNA methylation and colorectal cancer. Curr Colorectal Cancer Rep 10: 425-430, 2014.

4. Evers BM: Neurotensin and growth of normal and neoplastic tissues. Peptides 27: 2424-2433, 2006.

5. Kalafatakis K and Triantafyllou K: Contribution of neurotensin in the immune and neuroendocrine modulation of normal and abnormal enteric function. Regul Pept 170: 7-17, 2011.

6. Li J, Song J, Zaytseva YY, Liu Y, Rychahou P, Jiang K, Starr ME, Kim JT, Harris JW, Yiannikouris FB, et al: An obligatory role for neurotensin in high-fat-diet-induced obesity. Nature 533: 411-415, 2016.

7. Mustain WC, Rychahou PG and Evers BM: The role of neurotensin in physiologic and pathologic processes. Curr Opin Endocrinol Diabetes Obes 18: 75-82, 2011.

8. Wu Z, Martinez-Fong D, Trédaniel J and Forgez P: Neurotensin and its high affinity receptor 1 as a potential pharmacological target in cancer therapy. Front Endocrinol (Lausanne) 3: 184, 2013.

9. Dupouy S, Mourra N, Doan VK, Gompel A, Alifano M and Forgez P: The potential use of the neurotensin high affinity receptor 1 as a biomarker for cancer progression and as a component of personalized medicine in selective cancers. Biochimie 93: 1369-1378, 2011.

10. Wang L, Friess H, Zhu Z, Graber H, Zimmermann A, Korc M, Reubi JC and Büchler MW: Neurotensin receptor-1 mRNA analysis in normal pancreas and pancreatic disease. Clin Cancer Res 6: 566-571, 2000.

11. Souazé F, Dupouy S, Viardot-Foucault V, Bruyneel E, Attoub S, Gespach C, Gompel A and Forgez P: Expression of neurotensin and NT1 receptor in human breast cancer: A potential role in tumor progression. Cancer Res 66: 6243-6249, 2006.

12. Gui X, Guzman G, Dobner PR and Kadkol SS: Increased neurotensin receptor-1 expression during progression of colonic adenocarcinoma. Peptides 29: 1609-1615, 2008.

13. Dupouy S, Viardot-Foucault V, Alifano M, Souazé F, Plu-Bureau G, Chaouat M, Lavaur A, Hugol D, Gespach C, Gompel A, et al: The neurotensin receptor-1 pathway contributes to human ductal breast cancer progression. PLoS One 4: e4223, 2009.

14. Alifano M, Souazé F, Dupouy S, Camilleri-Broët S, Younes M, Ahmed-Zaïd SM, Takahashi T, Cancellieri A, Damiani S, Boaron M, et al: Neurotensin receptor 1 determines the outcome of non-small cell lung cancer. Clin Cancer Res 16: 4401-4410, 2010.

15. Valerie NC, Casarez EV, Dasilva JO, Dunlap-Brown ME, Parsons SJ, Amorino GP and Dziegielewski J: Inhibition of neurotensin receptor 1 selectively sensitizes prostate cancer to ionizing radiation. Cancer Res 71: 6817-6826, 2011. 
16. Kim JT, Li J, Song J, Lee EY, Weiss HL, Townsend CM Jr and Evers BM: Differential expression and tumorigenic function of neurotensin receptor 1 in neuroendocrine tumor cells. Oncotarget 6: 26960-26970, 2015

17. Kim JT, Li J, Jang ER, Gulhati P, Rychahou PG, Napier DL, Wang C, Weiss HL, Lee EY, Anthony L, et al: Deregulation of $\mathrm{Wnt} / \beta$-catenin signaling through genetic or epigenetic alterations in human neuroendocrine tumors. Carcinogenesis 34: 953-961, 2013.

18. Kim JT, Liu C, Zaytseva YY, Weiss HL, Townsend CM Jr and Evers BM: Neurotensin, a novel target of Wnt/ $\beta$-catenin pathway, promotes growth of neuroendocrine tumor cells. Int J Cancer 136: 1475-1481, 2015

19. Evers BM, Ishizuka J, Chung DH, Townsend CM Jr and Thompson JC: Neurotensin expression and release in human colon cancers. Ann Surg 216: 423-430; discussion 430-431, 1992.

20. Zhao D, Kuhnt-Moore S, Zeng H, Wu JS, Moyer MP and Pothoulakis C: Neurotensin stimulates IL-8 expression in human colonic epithelial cells through Rho GTPase-mediated NF-kappa B pathways. Am J Physiol Cell Physiol 284: C1397-C1404, 2003.

21. Ning Y, Manegold PC, Hong YK, Zhang W, Pohl A, Lurje G, Winder T, Yang D, LaBonte MJ, Wilson PM, et al: Interleukin-8 is associated with proliferation, migration, angiogenesis and chemosensitivity in vitro and in vivo in colon cancer cell line models. Int J Cancer 128: 2038-2049, 2011.

22. Kerkhoff E and Rapp UR: Cell cycle targets of Ras/Raf signalling. Oncogene 17: 1457-1462, 1998.

23. Servotte S, Camby I, Debeir O, Deroanne C, Lambert CA, Lapière CM, Kiss R, Nusgens B and Decaestecker C: The in vitro influences of neurotensin on the motility characteristics of human U373 glioblastoma cells. Neuropathol Appl Neurobiol 32: $575-584,2006$
24. Shimizu S, Tsukada J, Sugimoto T, Kikkawa N, Sasaki K, Chazono H, Hanazawa T, Okamoto Y and Seki N: Identification of a novel therapeutic target for head and neck squamous cell carcinomas: A role for the neurotensin-neurotensin receptor 1 oncogenic signaling pathway. Int J Cancer 123: 1816-1823, 2008.

25. Souazé F, Viardot-Foucault V, Roullet N, Toy-Miou-Leong M, Gompel A, Bruyneel E, Comperat E, Faux MC, Mareel M, Rostène $\mathrm{W}$, et al: Neurotensin receptor 1 gene activation by the Tcf/beta-catenin pathway is an early event in human colonic adenomas. Carcinogenesis 27: 708-716, 2006.

26. Dong Z, Wang X, Zhao Q, Townsend CM Jr and Evers BM: DNA methylation contributes to expression of the human neurotensin/ neuromedin N gene. Am J Physiol 274: G535-G543, 1998.

27. Dong Z, Wang X and Evers BM: Site-specific DNA methylation contributes to neurotensin/neuromedin $\mathrm{N}$ expression in colon cancers. Am J Physiol Gastrointest Liver Physiol 279: G1139-G1147, 2000.

28. Hagihara A, Miyamoto K, Furuta J, Hiraoka N, Wakazono K, Seki S, Fukushima S, Tsao MS, Sugimura T and Ushijima T: Identification of $275^{\prime} \mathrm{CpG}$ islands aberrantly methylated and 13 genes silenced in human pancreatic cancers. Oncogene 23: 8705-8710, 2004

29. Tan AC, Jimeno A, Lin SH, Wheelhouse J, Chan F, Solomon A, Rajeshkumar NV, Rubio-Viqueira $B$ and Hidalgo $M$ : Characterizing DNA methylation patterns in pancreatic cancer genome. Mol Oncol 3: 425-438, 2009.

30. Guo S, Yan F, Xu J, Bao Y, Zhu J, Wang X, Wu J, Li Y, Pu W, Liu Y, et al: Identification and validation of the methylation biomarkers of non-small cell lung cancer (NSCLC). Clin Epigenetics 7: 3, 2015. 